\title{
Fascículo Supernumerario del Músculo Abductor del Dedo Mínimo de la Mano. Importancia Anatómica y Clínica
}

\author{
Supernumerary Fascicle of the Abductor digiti minimi Muscle of the Hand. \\ Anatomical and Clinical Importance
}

"Mariano del Sol \& **Bélgica Vásquez

DEL SOL, M. \& VÁSQUEZ, B. Fascículo supernumerario del músculo abductor del dedo mínimo de la mano. Int. J. Morphol., 27(4):1069-1072, 2009.

RESUMEN: La existencia de músculos supernumerarios originados en el antebrazo, con un vientre muscular prolongándose hasta la mano, es infrecuente y por lo mismo, no existe mucha literatura sobre el tema, a pesar que estos músculos podrían producir síndromes de compresión a nivel del túnel carpiano o del túnel ulnar. Describimos la presencia de un fascículo supernumerario del músculo abductor del dedo mínimo, encontrado en el antebrazo y mano de un cadáver formolizado, de sexo masculino, adulto, disecado en la Unidad de Anatomía de la Facultad de Medicina de la Universidad de La Frontera, Chile. El músculo supernumerario destinado al dedo mínimo, se originaba por medio de fibras carnosas, en el tercio distal del antebrazo, desde el tendón de un músculo palmar largo accesorio. Con estrecha relación con los vasos y nervio ulnares, se insertaba junto a los músculos abductor del dedo mínimo, flexor corto del dedo mínimo y parte del oponente del dedo mínimo, en la falange proximal del dedo mínimo de la mano. Este fascículo muscular mantenía estrechas relaciones anatómicas, a nivel del carpo, con el nervio ulnar, pudiendo haber causado una eventual compresión de este nervio a su paso por el canal ulnar.

PALABRAS CLAVE: Anatomía; Mano; Músculos; Variación anatómica.

\section{INTRODUCCIÓN}

La presencia de músculos o fascículos supernumerarios, con origen en el antebrazo e inserción en la mano, no es frecuentemente encontrada en los textos de Anatomía y Cirugía. Su presencia puede, en algunos casos, provocar síndromes de compresión de los nervios mediano o ulnar.

Según Le Double (1897), un fascículo supernumario del músculo abductor del dedo mínimo de la mano fue descrito por primera vez por Soemmerring. Posteriormente, en el año 1868, Wood reportó que había encontrado en un sujeto, al músculo abductor del dedo mínimo constituido por dos partes, una de ellas reproduciendo al músculo normal y la otra remontándose hacia la muñeca. Este última parte, estaba subdividida en dos fascículos, el primero se originaba de la fascia que recubría al músculo flexor ulnar del carpo y el segundo del tendón del músculo palmar largo. Posteriormente, Wood disecó y encontró esta variación en 3/102 casos, siendo cada uno de estos hallazgos, con diferenets orígenes e inserciones.

\footnotetext{
* Facultad de Medicina, Universidad de La Frontera, Temuco, Chile.

** Universidad Autónoma de Chile, Sede Temuco, Chile.
}

Se ha relacionado a los músculos palmares largos accesorios o duplicidad del músculo palmar largo como causantes del síndrome de compresión del nervio mediano (Bang et al., 1988). Según el estudio de estos autores el nervio mediano en ambos lados fue comprimido por músculos anormales con origen en la parte medial del tendón del músculo palmar largo normal e inserción en la región hipotenar. Según estos autores, los síntomas clínicos desaparecieron por excisión de los músculos anormales. Regan et al., (1991) relataron la compresión del nervio y vasos ulnares por la presencia de este mismo músculo; sin embargo, señalaron que el músculo palmar accesorio se originaba de la base del quinto metacarpiano y se inserta en el tendón del músculo palmar largo.

Sañudo et al. (1993) describieron dos casos en que el músculo abductor del dedo mínimo presentaba fascículos anómalos. Ambos fascículos se originaban del retináculo flexor y de la fascia antebraquial, insertándose uno de ellos 
en el músculo abductor del dedo mínimo y el otro en la falange proximal del dedo mínimo. Según estos autores, las anomalías musculares cruzaban al nervio ulnar, y en un caso, también al nervio mediano.

Estas variaciones musculares también han sido observadas a través de la resonancia magnética. Zeiss \& Jakab (1995) demostraron la presencia de un músculo anómalo en un paciente que presentaba dolor focal e hinchazón en la muñeca y compresión de los nervios mediano y ulnar. El músculo se originaba del tendón del músculo palmar largo y de la fascia antebraquial, divergía medialmente del tendón del músculo palmar largo y se bifurcaba. Ambas porciones se extendían en el canal ulnar. Uno de los segmentos del músculo se unía con el músculo abductor del dedo mínimo y el otro con el retináculo flexor.

Una revisión de la compresión de los nervio ulnar y mediano, a nivel de la muñeca, causado por anomalías musculares fue realizada por se Smet (2002). Este autor señaló que al examinar el nervio ulnar en el canal ulnar (de Guyon) es posible encontrar anomalías musculares como músculo palmar anormal (reverso o accesorio), músculos hipotenares anormales (duplicación o inserción anormal) y un fascículo aberrante del músculo flexor ulnar del carpo y nomina a esta formación como complejo del músculo palmar largoabductor del dedo mínimo.

Kawashima et al. (2002) relatn que durante una disección, observaron la duplicación del músculo palmar largo y un músculo palmar largo accesorio, todos originados del epicóndilo medial del húmero, localizándose los tres músculos aberrantes entre los músculos flexor ulnar del carpo y flexor radial del carpo y terminando uno de ellos en la aponeurosis palmar y los otros dos en el retináculo flexor.

Durante una disección Bozkurt et al. (2004) encontraron que el músculo palmar largo, terminaba dividiéndose en dos tendones: uno se insertaba en la aponeurosis palmar y el otro en la parte proximal del retináculo flexor. Adicionalmente, encontraron un músculo accesorio que se extendía entre el retináculo flexor y el tendón del músculo abductor del dedo mínimo. Este músculo se localizaba profundo a la arteria ulnar pero superficial a los ramos superficial y profundo del nervio ulnar a nivel de la muñeca.

Por la importancia que tiene la presencia de los músculos supernumerarios a nivel del antebrazo y mano, especialmente cuando se encuentran dentro un canal anatómico estrecho e inelástico como el canal ulnar, creemos que el comunicar esta variación anatómica, contribuye al conocimiento anatómico y clínico de las regiones involucradas.

\section{DESCRIPCIÓN}

Describimos una variación anatómica muscular encontrada durante la disección del antebrazo y mano en un cadáver formolizado, de sexo masculino, adulto, perteneciente a la Unidad de Anatomía de la Facultad de Medicina de la Universidad de La Frontera, Chile.

$\mathrm{Al}$ seccionar la fascia antebraquial, en la parte distal de la región anterior del antebrazo, observamos los tendones de dos músculos palmares largos: lateral y medial (una variación anatómica frecuente) y al tendón del músculo flexor ulnar del carpo. De la cara anterior del tendón del músculo palmar largo accesorio (medial) se observó el origen de un músculo supernumerario, que denominamos fascículo accesorio del músculo abductor del dedo mínimo (FAMADM). Fig. 1.

El FAMADM, unnipennado, tenía su origen en el tercio distal del antebrazo, en el tendón del músculo palmar largo accesorio (medial) y en la fascia antebraquial profunda . El origen del músculo tenía aproximadamente $25 \mathrm{~mm}$ y sus fibras musculares se dirigían medial y distalmente hacia la mano. Las fibras musculares en su descenso se contorneaban, colocándose las más mediales profundas al tendón del músculo flexor ulnar del carpo. A medida que descendía y entraba a la mano, el cuerpo muscular se tornaba más cilíndrico, colocándose lateral a los huesos pisiforme y hamato (separado de estas estructuras por los vasos ulnares y nervio ulnar), lugar de origen de los músculos abductor del dedo mínimo y flexor corto del dedo mínimo, respectivamente. (Fig. 2).

$\mathrm{Al}$ penetrar en la mano, el FAMADM se relacionaba: anteriormente con el músculo palmar corto, posteriormente con la aponeurosis palmar y medialmente con el hueso pisiforme, la inserción tendinosa del músculo flexor ulnar del carpo y el ligamento pisiuncinado. Luego, las fibras del FAMADM, se colocaban lateral al músculo abductor del dedo mínimo, anterior y luego anteromedial al músculo flexor corto del dedo mínimo, al cual cruzaba, y anteromedial al músculo oponente del dedo mínimo. Finalmente, las fibras musculares, FAMADM se unieron a las fibras del músculo abductor del dedo mínimo (Figs. 3 y 4). Las fibras musculares de los tres músculos: abductor del dedo mínimo, FAMADM y flexor corto del dedo mínimo, se unían en un tendón, el cual se insertaba en la falange proximal del dedo mínimo. Además, cerca de su inserción y por su parte lateral, algunas fibras del músculo oponente del dedo mínimo se unían a la inserción del FAMADM.

El FAMADM, durante su descenso era cruzado por 


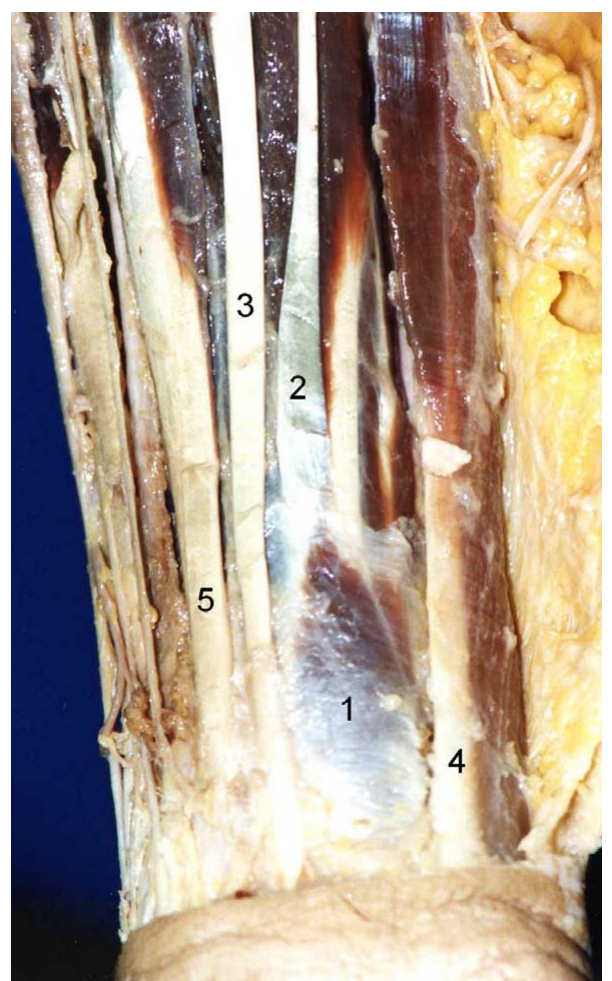

Fig. 1. Región ventral del antebrazo. 1. Fascículo supernumerario del $\mathrm{m}$. abductor del dedo mínimo de la mano. 2 y 3 . Tendones de los mm. palmares largos normal y accesorio (medial); 4 . Tendón del $\mathrm{m}$. flexor ulnar del carpo; 5. Tendón del m. flexor radial del carpo.

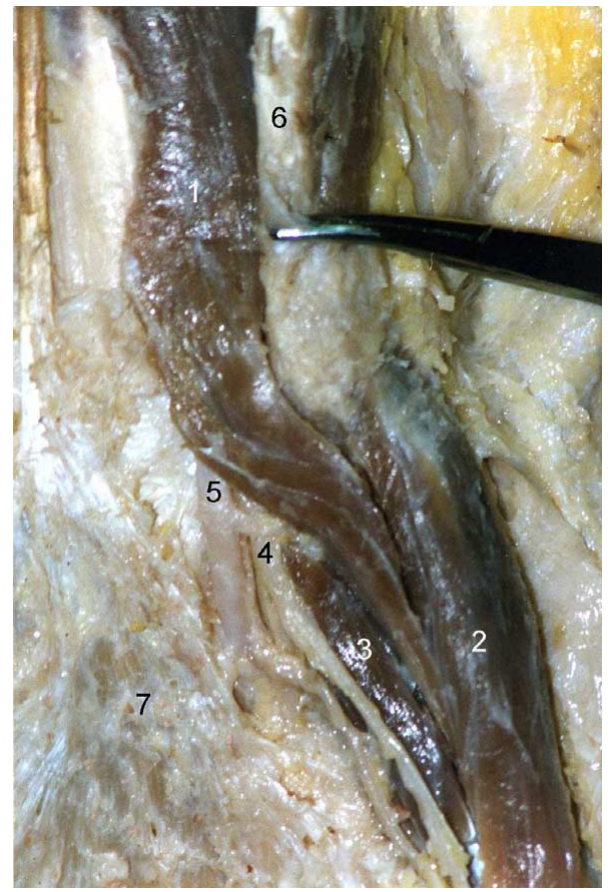

Fig. 3. Región palmar. 1. Fascículo supernumerario del $\mathrm{m}$. abductor del dedo mínimo de la mano; 2 . M. abductor del dedo mínimo; 3 . M. flexor corto del dedo mínimo; 4. Nervio ulnar; 5. Arteria ulnar; 6. Tendón del m. flexor ulnar del carpo; 7 Aponeurosis palmar.

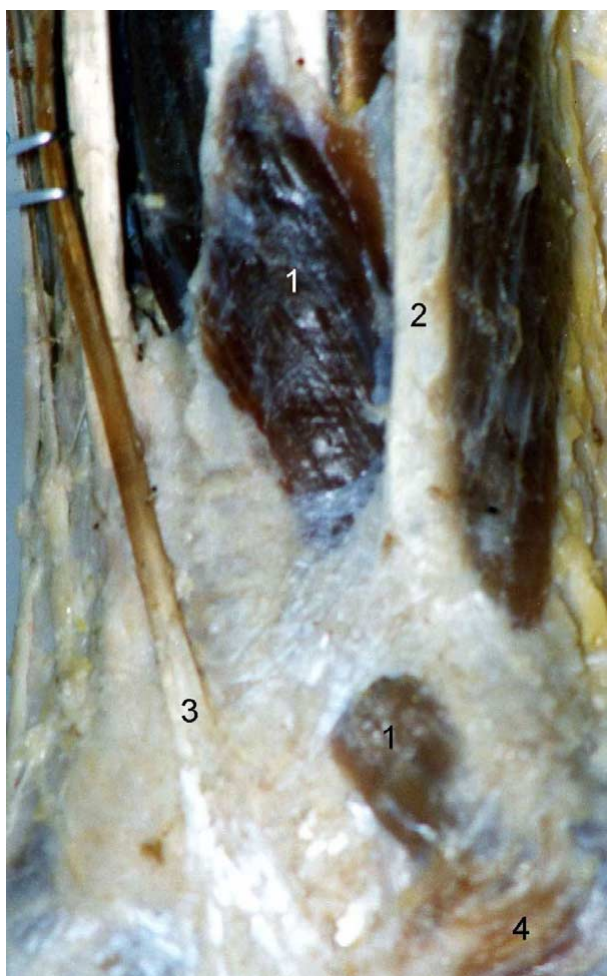

Fig. 2. Región ventral del tercio inferior del antebrazo. 1. Fascículo supernumerario del $\mathrm{m}$. abductor del dedo mínimo de la mano; 2 . Tendón del $\mathrm{m}$. flexor ulnar del carpo; 3. Tendón del m. palmar largo; 4. M. palmar corto.

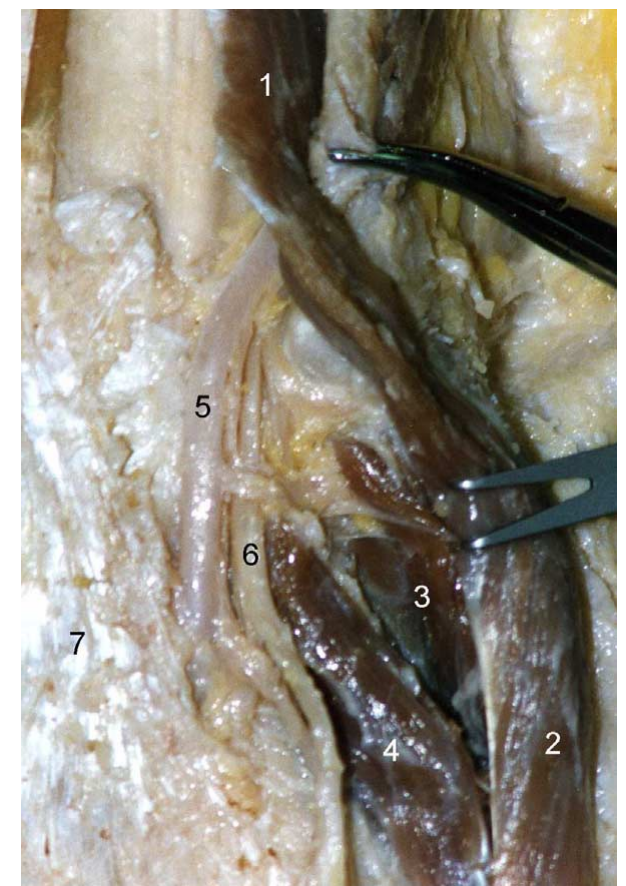

Fig. 4. Región palmar. 1. Fascículo supernumerario del $\mathrm{m}$. abductor del dedo mínimo de la mano; 2. M. abductor del dedo mínimo; 3. M. flexor corto del dedo mínimo; 4. M. oponente del dedo mínimo; 5. Arteria ulnar; 6. Nervio ulnar; 7. Aponeurosis palmar. su cara posterior por los vasos ulnares y el nervio ulnar (Fig. 4). Al inicio, el nervio ulnar se localizaba medial al músculo, luego posterior y finalmente inferolateral al FAMADM. La arteria ulnar siempre se mantuvo lateral al nervio ulnar.

\section{DISCUSIÓN}

Síndrome de compresión del nervio ulnar en el canal ulnar puede ser de variada etiología; sin embargo, no resulta frecuente encontrar en la literatura científica, muchos relatos sobre causantes musculares de esta patología. A pesar de lo anterior, Shea \& McClain (1969) señalaron que el 2,9\% de los casos de síndrome de compresión del nervio ulnar se debe a la presencia de un músculo aberrante.

Swanson et al. (1972) presentaron un caso bilateral de compresión del nervio ulnar a nivel de la muñeca causado por un músculo anómalo en el canal ulnar. Estos autores señalaron el trayecto y relaciones muy similares a nuestro caso, pero con una inserción en el músculo flexor corto del dedo mínimo, el cual además de provocar la compresión nerviosa, su tracción produciría una flexión del dedo mínimo, a nivel de la articulación metacarpofalángica. 
El origen de un fascículo adicional o FAMADM puede ser una variación o una anomalías dependiendo si altera o no la función muscular, o es causa de un síndrome de compresión nerviosa. Estas formaciones, como lo señalan Sañudo et al., reciben numerosos nombres, tales como abductor largo del quinto dedo, músculo flexor corto accesorio del dedo mínimo, accesorio y abductor de dedo mínimo de la mano. Para algunos autores, estos fascículos musculares son considerados como accesorios del músculo palmar largo y no del músculo abductor del dedo mínimo.

Creemos poco probable que ocurra lo señalado por Regan et al., quienes indicaron que el músculo palmar accesorio se originaba de la base del quinto metacarpiano y se insertaba en el tendón del músculo palmar largo. Esto nos llevaría a sustentar un cambio en la mecánica articular, ya que estarían invertidos los puntos de origen e inserción y, por lo tanto, la manera de traccionar sería distinta. Podría tratarse más bien de un caso de músculo palmar reverso.

Músculos supernumerarios relacionados estrechamente con nervios y cruzando junto a ellos en sitios inelásticos, como el túnel ulnar, pueden provocar síndromes de compresión. En la actualidad, métodos no invasivos pueden determinar con exactitud la presencia de anomalías o variaciones musculares. Conocer estas variaciones musculares es importante para el anatomista, clínico y cirujano, quienes deben intervenir en la regiones de la muñeca y de la mano.

DEL SOL, M. \& VÁSQUEZ, B. Supernumerary fascicle of the Abductor digiti minimi muscle of the hand. Anatomical and clinical importance. Int. J. Morphol., 27(4):1069-1072, 2009.

SUMMARY: The existence of the supernumerary muscles originated in the forearm, with the muscular belly prolonging to the hand is infrequent. Therefore, there is not much literature available regarding this subject, despite that these muscles could produce symptoms of compression at the carpal tunnel or ulnar tunnel level. We describe the presence of a supernumerary fascicle of the abductor digiti minimi muscle, located in the forearm and hand of a formolized adult male cadaver, dissected at the Anatomy Unit of the Faculty of Medicine of the Universidad de La Frontera, Chile. The supernumerary muscle destined to the digiti minimi, originated by means of carnous fibers in the distal tertium of the forearm from the tendon of an accessory palmaris longus muscle. Closely related with the ulnar vessels and ulnar nerve, it inserted along with the abductor the digiti minimi muscle, flexor digiti minimi brevis muscle and part of the opponens digiti minimi muscle, in the proximal phalanx digiti minimi of the hand. This muscular fascicle maintained a close anatomical relation at the carpal level, with the ulnar nerve, possibly having caused an eventual compression of this nerve on its passage through the ulnar canal.

KEY WORDS: Anatomy; Hand; Muscles; Anatomical Variation.

\section{REFERENCIAS BIBLIOGRÁFICAS}

Bang, H.; Kojima, T. \& Tsuchida, Y. A case of carpal tunnel syndrome caused by accessory palmaris longus muscle. Handchir. Mikrochir. Plast. Chir., 20(3):141-3, 1988.

Bozkurt, M. C.; Tagil, S. M.; Ersoy, M. \& Tekdemir, I. Muscle variations and abnormal branching and course of the ulnar nerve in the forearm and hand. Clinical Anatomy., 17:64-6, 2004.

de Smet, L. Median and ulnar nerve compression at the wrist caused by anomalous muscles. Acta Orthopaedica Belgica., 68(5):431$8,2002$.

Kawashima, T.; Kikushima, S.; Yokota, E.; Ohkubo, F.; Yamana, Y.; Sato, F. \& Sasaki, H. A case of an accessory palmaris longus muscle and a duplicate palmaris longus muscle with special reference to their nerve supply. Okajimas Folia Anat., Jpn., 79(2-3):75-82, 2002.

Le Double, A. F. Traité des variations du systeme musculaire de l'homme. Paris, Librairie C. Reinwald Schleucher Frères, Editeurs, 1897. V. 2.

Regan, P. J.; Feldberg, L. \& Bailey, B. N. Accessory palmaris longus muscle causing ulnar nerve compresion at the wrist. The journal of Hand Surgery, 16(4):736-8, 1991.
Sañudo, J. R.; Mirapeix, R. M. \& Ferreira, B. A rare anomaly of abductor digiti minimi. J. Anat., 182:439-42, 1993.

Shea, J. D. \& McClain, E. J. Ulnar nerve compression syndromes at and below the wrist. J. of bone and Joint Surgey, 51A:1095102, 1969.

Swanson, A. B.; Biddulph, S. L.; Baughman, F. A. Jr. \& de Groot, G. Ulnar nerve compression due to an anomalous muscle in the canl of Guyon. Clinical orthopaedic and related research, 83:64-9, 1972.

Wood, J. Variations in human myology observed during the winter season of 1867-68 at King's College, London. In variations in human myology. Proceedings of the Royal Socciety of London, 16:483-525, 1868.

Zeiss, J. \& Jakab, E. MR demonstration of an anomalous muscle in a patient with coexistent carpal and ulnar tunnel syndrome. Case report and literature summary. Clin. Imaging, 19(2):1025,1995 .

Dirección para correspondencia:

Prof. Dr. Mariano del Sol

Facutad de Medicina

Universidad de La Frontera

Casilla 54-D

Temuco - CHILE

Email:mdelsol@ufro.cl

Recibido : 07-09-2009 Aceptado: 12-11-2009 